\title{
The design of a form-changing female fitting robot
}

\author{
Zengrong GUO*, Dongliang ZHANG*, Shaodong ZHANG*, Xingsuo LIU* and Jituo LI** \\ * International Design Institute, Zhejiang University \\ No. 388, Yuhangtang Road, Xihu District, Hangzhou 310058, China \\ E-mail: dzhang@zju.edu.cn \\ ${ }^{* *}$ Department of Mechanical Engineering, Zhejiang University \\ No. 38, Zheda Road, Xihu District, Hangzhou 310058, China
}

Received 25 January 2016

\begin{abstract}
We present a design of a form-changing female robotic mannequin for garment fitting. The fitting robot can change itself to imitate the body shape of a female person and replace her to finish the fitting process remotely. It can be used for reducing the return rate of garment e-commerce and increasing the order quantity of made-to-measure by avoiding the geographical restriction and the complicated process of trying on different clothes personally for a customer. In this paper, we mainly focus on the design of the mechanical structure and control system for the female fitting robot. By analyzing the characteristics of the skeleton structure and shape of female bodies, we design a block model for representing the surface of the fitting robot and an internal motion mechanism which is driven by a control system after inputting parameters of body sizes. On the basis of the design, a prototype of the form-changing female fitting robot is developed. Experimental results show that it can imitate a range of female body types.
\end{abstract}

Key words : Form-changing fitting robot, Remote fitting, Garment e-commerce, Made-to-measure, Mechanical structure, Control system, Prototype

\section{Introduction}

Nowadays, with the rapid development of Internet retailing and due to its convenience, more and more consumers prefer to online shopping. Clothing is a significant part of the Internet retailing and it is facing a serious problem of increasingly high rates of return goods. The main reason is that people could not try on the garment personally when they buy it online. Furthermore, the production standards of the clothing are different depending on the manufacturers. That makes it very difficult for customers to buy fitting clothes online. So it causes some consumers have to return the clothing more than once if they do not get the sizing they want. It makes that online shopping has become costly and time-consuming. In order to solve this problem, we design a form-changing female robotic mannequin. The consumers only need to input the data of body size on the user interface. Then the fitting robot can change itself to imitate the body shape of the consumer and carry out the complicated process of trying different clothes. Then the fitting results will be sent back to consumers in the form of photographs. It will enable consumers to see whether the clothes fit them and therefore, make better decisions. The fitting process of the fitting robot is shown in Fig. 1.

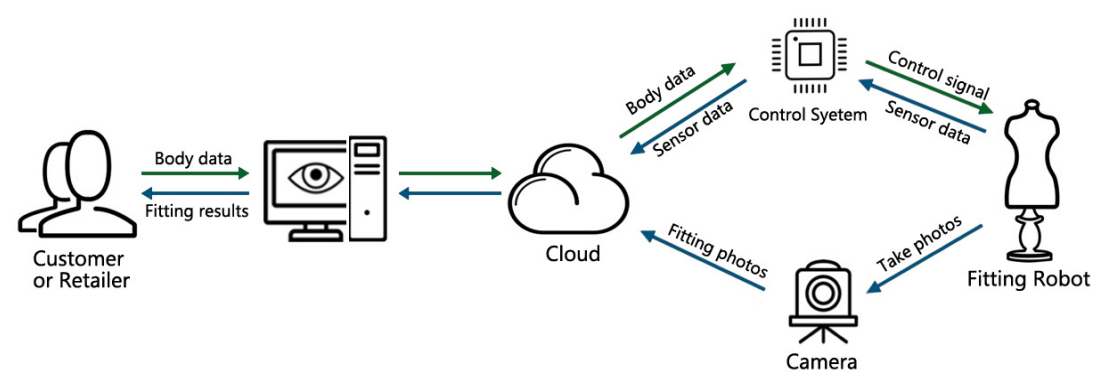

Fig. 1 The service process of a fitting robot. 
Made-to-measure garment is a kind of consumer goods for high-income groups. In the process of made-to-measure, consumers have to try on the garment for several times to ensure whether it is suitable for them. It requires the customer and the tailor to be located geographically near to each other. So the geographical position is a main factor that restricts the development of the made-to-measure garment industry. The design of the fitting robot solves this problem. Consumers only need to input their size data into the fitting robot. Then the fitting robot can imitate the body shape of the consumers and finish the fitting process for several times. This feature allows consumers to customize their own clothes wherever they are.

In our study, we introduce a female fitting robot, which is mainly used for garment e-commerce and made-to-measure. We focus on the design of its mechanical structure and control system. The robot can change its neck circumference, shoulder width, shoulder thickness, chest circumference, chest type, waist circumference, hip circumference, chest-waist height and waist-hip height.

\section{Related work}

Currently there are several companies and research institutions dedicated to the design of humanoid fitting robots that consist of precise mechanical structures and advanced control systems. One of the most prominent fitting robots is Fitbot (Abels and Kruusmaa, 2013a) developed by Fits.me company (Homepage, accessed on 11 May, 2015) in Estonia. The mechanical structure and control system of the robot are very complicated (Abels and Kruusmaa, 2009). It uses 50 drivers to simulate the bone and soft tissue, and it can simulate the shape of most people (Abels and Kruusmaa, 2013b). But the robot only has the upper body model and the price is very expensive, so it has not been widely used. The Hong Kong Polytechnic University designed an interactive robotic mannequin for the fashion industry (Peng, 2013). The robot has 20 degrees of freedom. Its deformation precision is $0.1 \mathrm{~cm}$ and the deformation velocity is $8.1 \mathrm{~s}$. But the quantity of shell panels is small, so the robot can only simulate limited human body types.

With the development of digital technology, virtual reality technology and Internet technology, people begin to use virtual fitting technology to solve the problems of fitting. At present, the main form of virtual fitting is the virtual fitting system and virtual fitting mirror. Virtual fitting techniques require the generation of 3D models of garments, which is costly and the product cycle is very long. Furthermore, due to the limitation of the technologies of 3D garment modeling, physical simulation and rendering, it is difficult to realistically display the texture and the wearing effect of clothing fabrics. Because of the variety, complexity and irregularity of fabrics, modeling and simulation of the fabrics are very difficult. After the efforts of many experts and scholars, a series of fabric modeling methods (Choi and Ko, 2002, Terzopoulost et al., 1987, Weil, 1986) and simulation methods (Baraff and Witkin, 1998, Goldenthal et al., 2007, Lee et al., 2010, Zhou et al., 2008) have been proposed, and a number of virtual fitting products are developed, such as the virtual fitting website of Metail Company (Homepage, accessed on 13 May, 2015) and the virtual fitting room of AR Door company (Homepage, accessed on 19 May, 2015). The existing virtual fitting products for parts of the fabric simulation can achieve a vivid visual effect, but there is still a big gap when it is compared with real cloth. Therefore, it is also difficult for the 3D virtual fitting technology to become the mainstream of the fitting method.

Compared with the virtual fitting technology, the fitting effect of the fitting robot is much more realistic and it has more resemblance to actual fitting. The fitting robot responds quickly and can be repeatedly used for a long time. It can also combine with the service of virtual fitting. So it will have bigger development space and application in the fashion industry.

\section{Initial model and panels design}

The surface of the fitting robot is consisted of a number of panels. To design the surface panels, we need to create an initial body model. In order to guarantee that the fitting robot has a reasonable deformation range, we choose one mannequin of the middle size from standard mannequins. The mannequin will be scanned for creating the initial model. We use the parametric modeling method (Kim and Park, 2004) and feature-based modeling method (Wang, 2005) to generate a 3D human model. A polyhedral modeling method (Baek and Lee, 2012) is used to approximate the surface with small triangular patches. The final model is represented by a triangular mesh.

After obtaining the initial human model, we design the model panels by analyzing the distribution of human muscles and characteristics of morphology deformation. The result is shown in Fig. 2. In order to make the 
morphological changes of the robot smooth, we divide the parametric model surface into 25 panels. Compared with the 16 panels of the interactive robotic mannequin that designed by Hong Kong Polytechnic University, the fitting robot can simulate the female body better. There is a gap of $1 \mathrm{~cm}$ between the panels. The gap provides space when the fitting robot deforms to small sizes. The design of panels tries to eliminate the possible steps between the panels and achieve the smooth transition between panels.
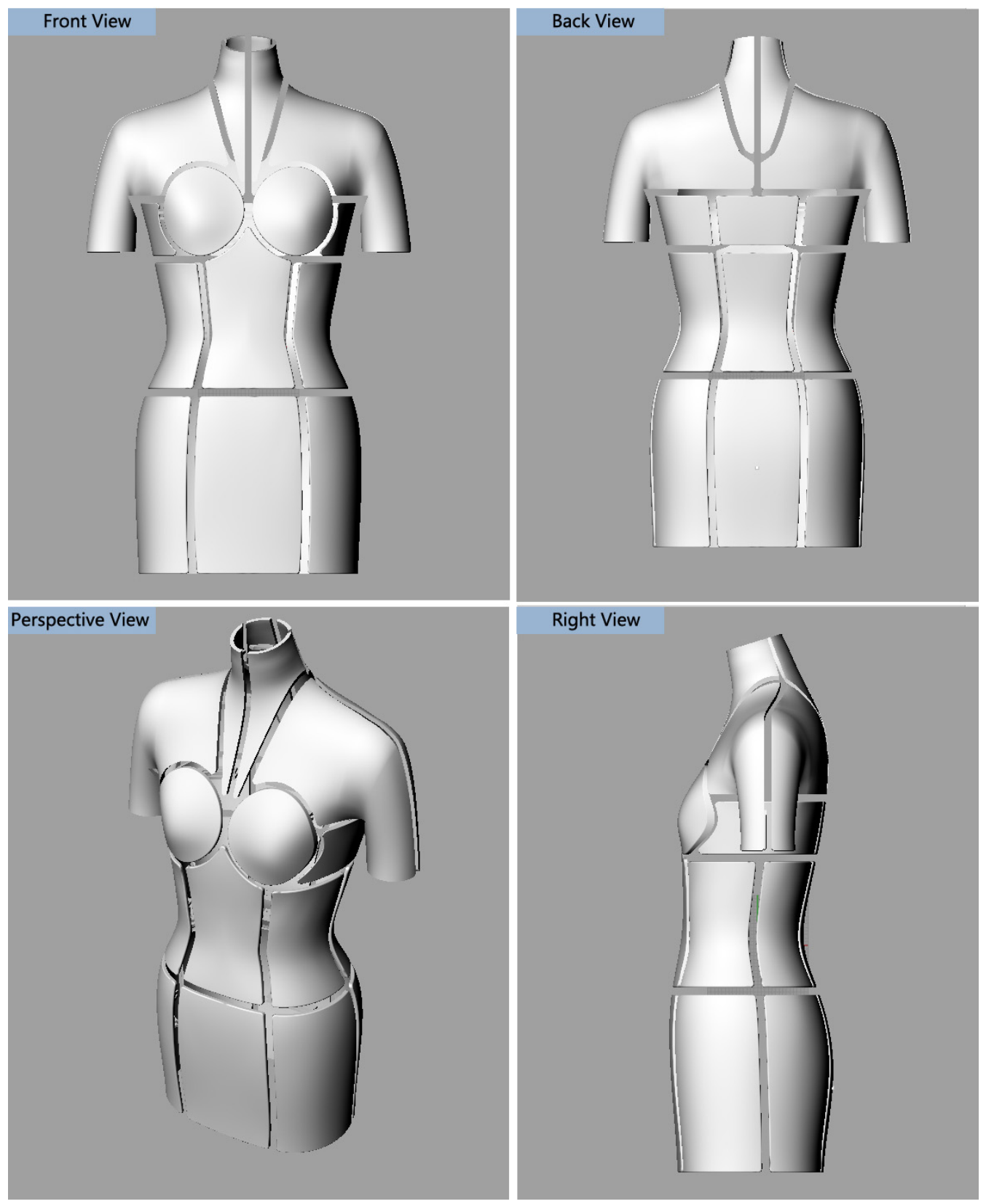

Fig. 2 Surface panels of the fitting robot.

\section{Mechanical structure design}

The difficulty in the design of the mechanical structure is to put the whole complex mechanism in a narrow internal space of the female body and the mechanism requires a certain range of motion. In addition, it is required to have a certain amount of thrust margin to deal with the resistance of clothing on the shell. And it also needs to design an installation structure to connect the shell panels and the internal mechanism.

The design of mechanical structure is a core part of the fitting robot. It is mainly used to complete the following tasks: 
(1) A certain size range of human bodies are simulated according to the driven data. The important and adjustable body parameters include neck circumference, shoulder width, shoulder thickness, chest circumference, chest type, waist circumference, hip circumference, chest-waist height and waist-hip height.

(2) Under the driving of stepper motors, the size of the robot is changed by moving the panels through a variety of connecting rods, gear racks, sliders and other mechanisms. The change rates of the front part and the back part of the human body are different (Taylor and Shoben, 1993), so the two parts of the mechanical structure are driven separately.

(3) The preset range of deformation is achieved through the structural design. After realizing the basic functions, the internal mechanism is simplified as far as possible to reduce the cost of the whole structure.

We design the mechanical structure of each layer according to the available space, the movement direction of pushrod and the range of motion. Then we assembly the parts using Solidworks software and ensure the practicality of each structure through finite element analysis. After that, we carry out interference inspection of virtual assembly to ensure the effective installation between the mechanical structure and the panels. The assembly results of mechanical structures of all layers are shown in Fig. 3- 7.

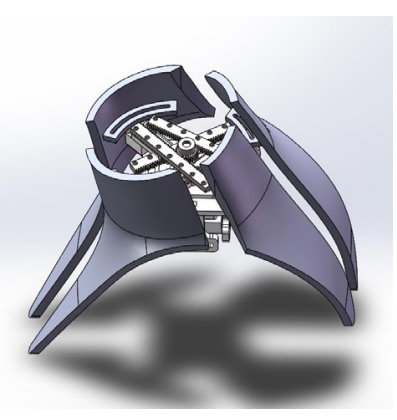

Fig. 3 Neck assembly

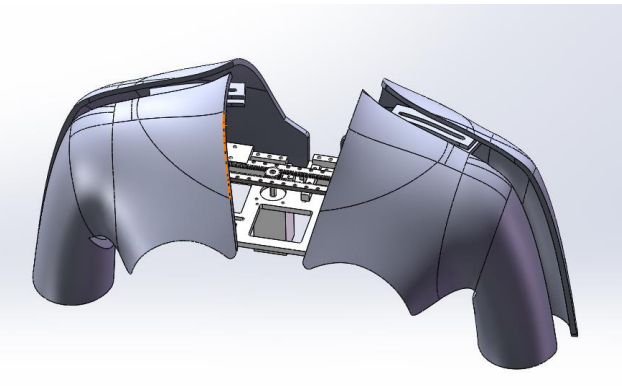

Fig. 4 Shoulder assembly

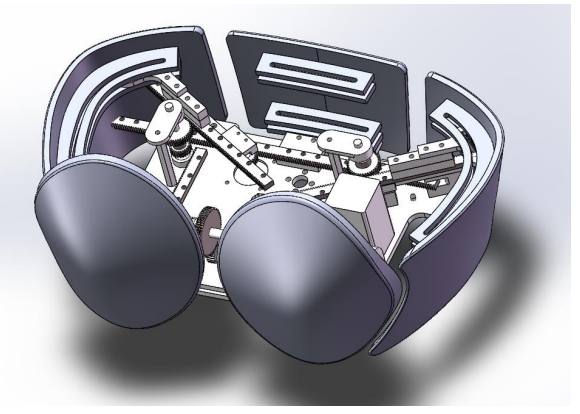

Fig. 5 Chest assembly

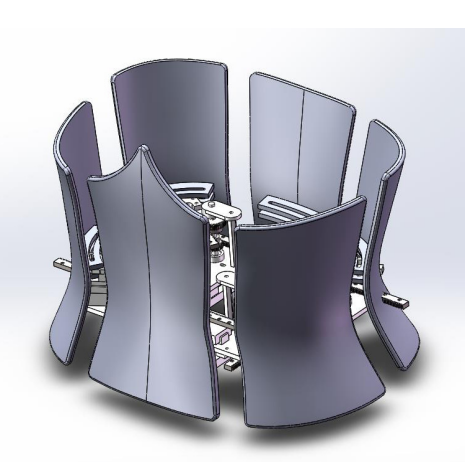

Fig. 6 Waist assembly

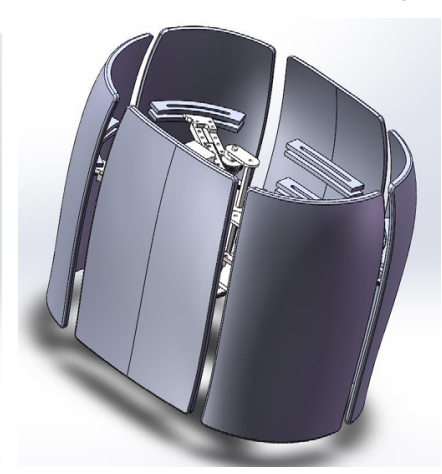

Fig. 7 Hip assembly

The fitting robot consists of five installation platforms. The motion of the pushrod can be decomposed into linear motion in several directions and unique rotary motion of the chest. The transmission of the gear and the screw rod is stable and the transmission ratio is accurate. The horizontal line movement is driven by the gear, and it is combined with sliders to achieve the motor output. The radial linear movement is driven by the screw rod, and the rotary motion is achieved by the gear shaft.

Take the shoulder as an example to introduce how the mechanism achieves deformation. The main parameters of the shoulder are the width and thickness. Therefore, we decompose the movement of the shoulder mechanism into $\mathrm{X}$ and Y directions. The change rates of the front part and the back part of the shoulder have little difference (Cheverud et al., 1988), so the two parts of the shoulder mechanism are driven simultaneously. The shoulder structure is shown in Fig. 8. Motor 1 starts to rotate and drive rack 1 and 2 to move in the opposite direction after receiving the instruction from the control system. The racks connect with the second stage installation platforms and sliders. The second stage installation platform 1 and 2 move to the left and right direction respectively under the action of racks and sliders. Then the width of the shoulder is changed. Motor 2 starts to rotate and drive rack 3 and 4 to move in the opposite direction after receiving the instruction from the control system. The pushrods move along the front and back direction respectively under the action of the racks and the sliders, then the thickness of the shoulder is changed. 


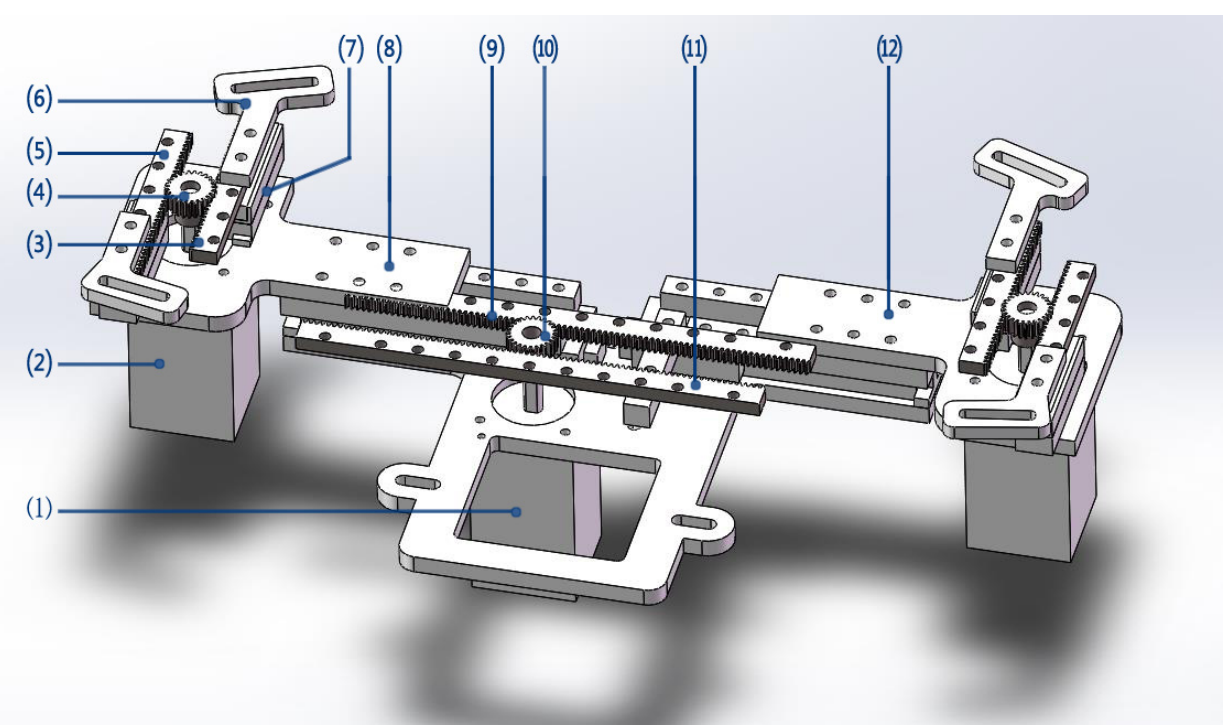

Fig. 8 Mechanical structure of the shoulder part (1) motor 1, (2) motor 2, (3) rack 3, (4) gear 2, (5) rack 4, (6) pushrod, (7) slider, (8) Second stage installation platform 1, (9) rack 1, (10) gear 1, (11) rack 2, (12)Second stage installation platform 2

The shell panels and the mechanical structure of the waist are divided into six parts. As the deformation range of the front part of the waist is much larger than the back part, the two parts of the mechanical structure are driven separately. The structure of the waist is shown in Figure 9. The angle between two pushrods is 60 degrees and the panels are mounted on the end of the pushrods. Each motor receives instructions from the control system at the same time. Motor 1 begins to rotate and drive rack 1 to move after receiving the instruction and rack 1 drive gear 2 and 3 to move. By installing rack 2 and 3 on the different sides of the gears, they can move forward or backward at the same time. Pushrod 1 and 2 move in different directions under the action of racks and the sliders, and the angle between the movement directions is 120 degrees. Motor 2 begins to rotate and drive rack 4 to move after receiving the instruction. Pushrod 3 and rack 4 move along the front and back direction under the action of sliders, so it will change the shape and size of the front part of the waist. The mechanical structure of the back part of the waist moves in the same way as the front part. Thus, the deformation of the whole waist can be achieved.

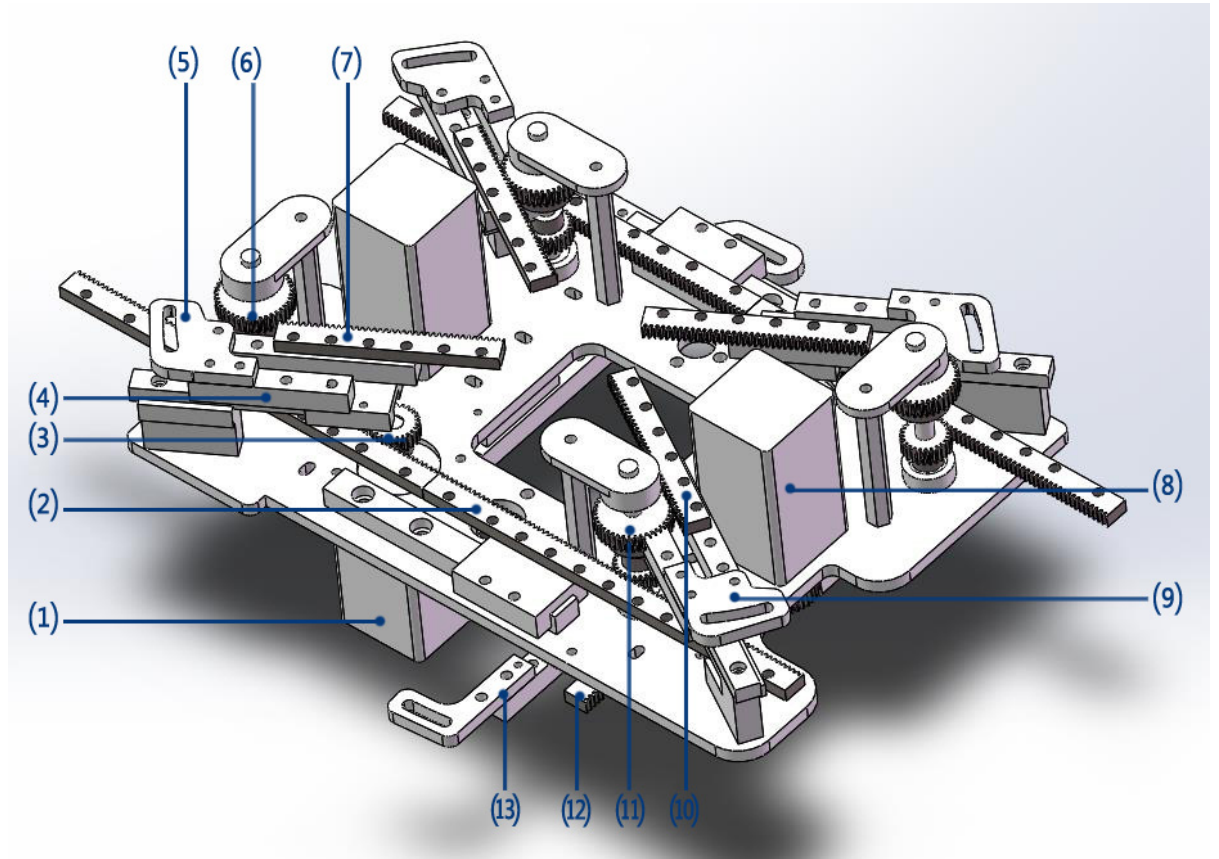

Fig. 9 Mechanical structure of the waist part (1) motor 1, (2) rack 1, (3) gear 1, (4) slider, (5) pushrod 1,
(6) gear 2, (7) rack 2, (8) motor 2,
(9) pushrod 2,
(10) rack 3 ,
(11) gear 3 ,
(12) rack 4, (13) pushrod 3 
The movement ways of mechanical structures of other parts are similar to the waist. After testing, the mechanical structure of the robot runs stably under the driven of the control system, and the simulation of the female human body can be realized.

\section{Control system design}

The control system is responsible for converting the data of body sizes that are input by users into instructions, and accurately controlling the movement of the mechanical structure to achieve the deformation of the robot. The control system of the fitting robot is shown in Fig. 10. The user inputs the data of body sizes through a control interface. Then the controller transforms the data into instructions that can be received by the motors. After receiving the instructions, the motors drive the mechanical structure to realize the deformation of the robot.

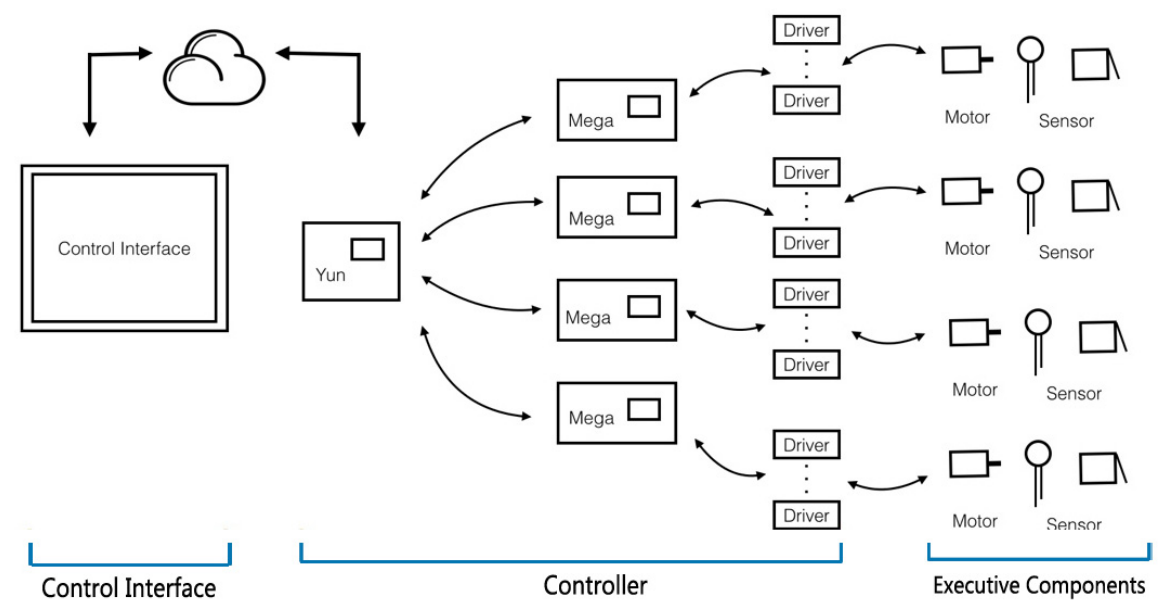

Fig. 10 Flow chart of the control system

For the design of the control system, we mainly consider the following aspects.

(1) More parameters need to be controlled in order to make the robot body more like the real one. The left and right structures can be driven by the same motor, so we use 19 motors to control 26 modules.

(2) Adopt a PCB circuit board to integrate Wi-Fi and Ethernet module to realize their communication, so that the user can control the fitting robot wirelessly. The design of the PCB circuit is shown in Fig. 11.

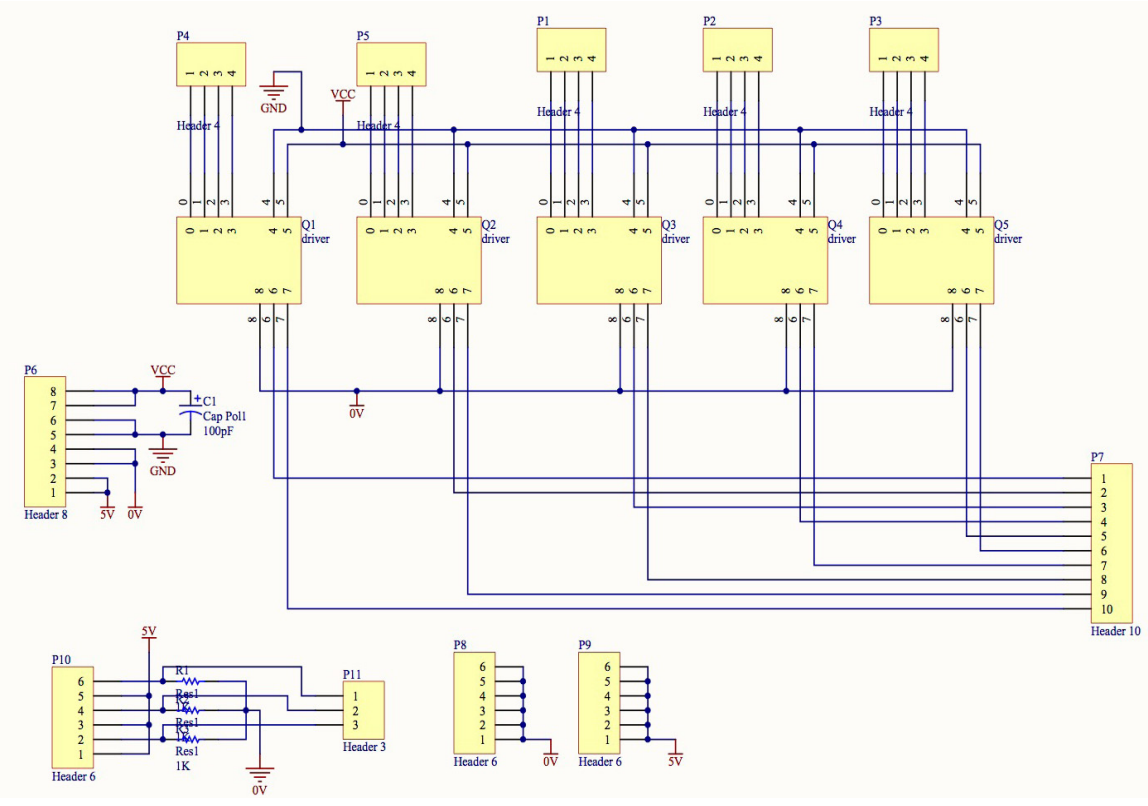

Fig. 11 The design of PCB circuit board 
(3) Signal communication is an important part of the control system, which includes communication between circuit boards, circuit boards and host computers, and communication between the wireless control platform and network control platform.

As for software, we use processing to develop the complete set of the control system. We employ SSH communication package of Java to realize the communication of Processing and the Wi-Fi module of Arduino, and use the cloud platform to set up online applications which can send control request of HTTP to Arduino to control the movement of the robot structure.

A control interface is designed to display the deformation effect of the robot in real time. Once the user inputs the body sizes, the 3D body model will be deformed. Meanwhile, the control system converts the size data into instructions which will be sent to the motors. Then, the motors drive the mechanical structures to realize the deformation of the robot to simulate the human body.

\section{Prototype}

Based on the design of hardware and software, we produce a prototype of the female fitting robot. The fitting robot can change the neck circumference, shoulder width, shoulder thickness, chest circumference, chest type, waist circumference, hip circumference, chest-waist height and waist-hip height. Compared with the interactive robot mannequin designed by Hong Kong Polytechnic University, the shell panels of fitting robot are increased by 9 and the deformation time is reduced by $3 \mathrm{~s}$. The deformation transition is smoother and the mechanical structure is more complex.

The prototype of the fitting robot can simulate a certain range of female body types. Fig. 12 shows the fitting robot of two different sizes. Fig. 13 shows the fitting results of different sizes for a T-shirt. In the figure, we can see that the T-shirt has the different shape when the sizes of the fitting robot are changed.

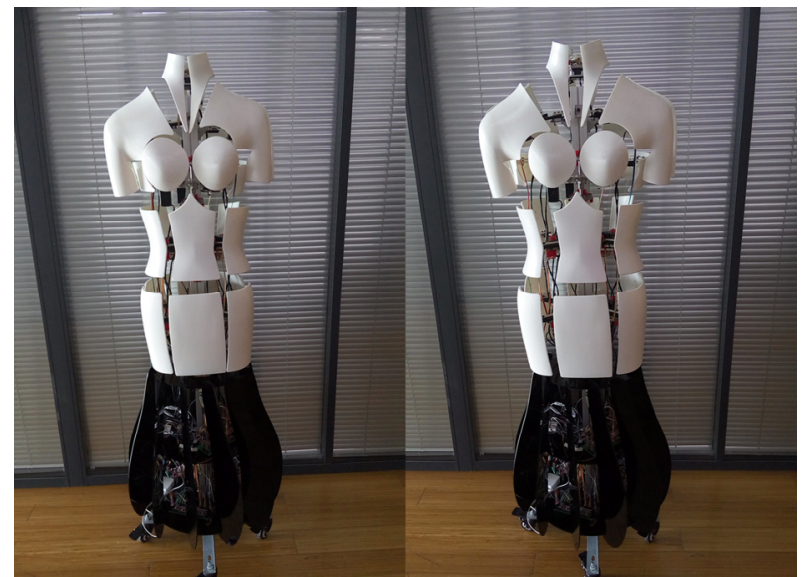

(a) Front view

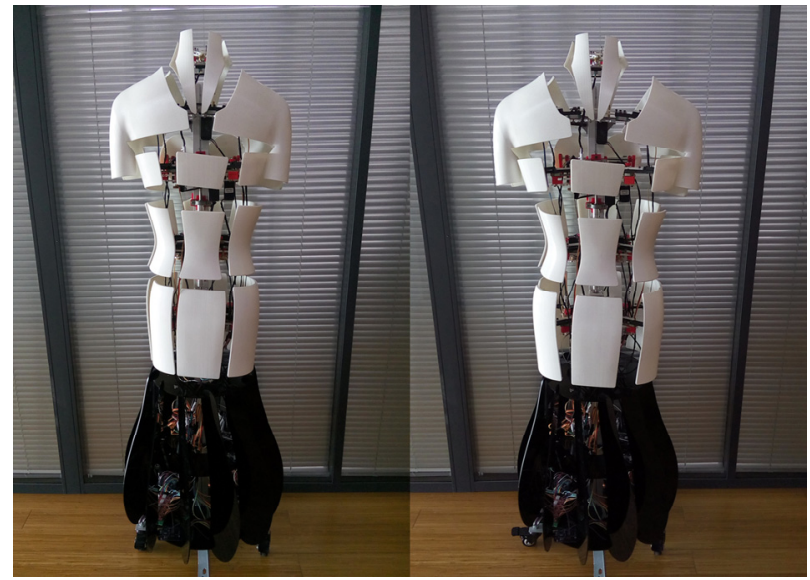

(b) Back view

Fig. 12 Fitting robot of two different sizes
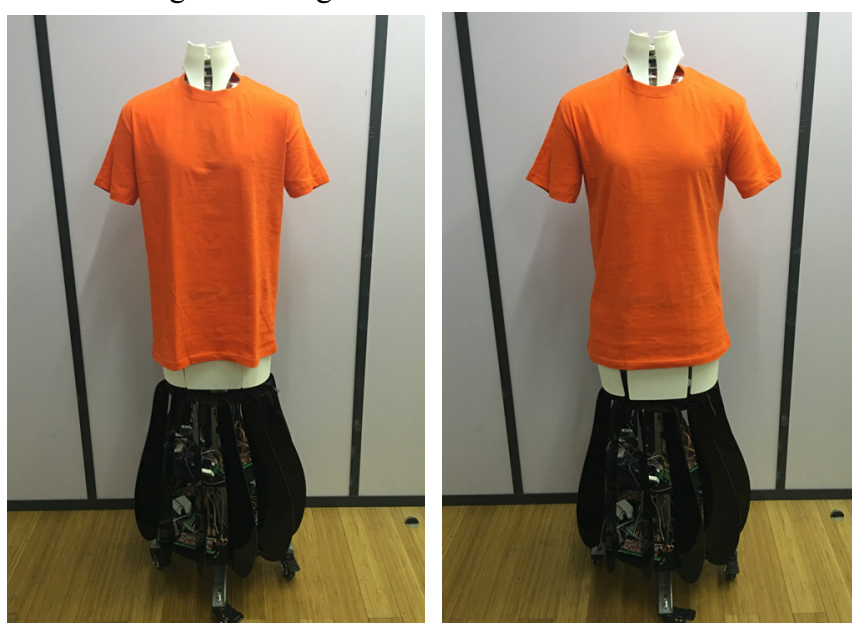

Fig. 13 Fitting results of different sizes 
Currently the prototype is in its early stage. Some limitations still exist. Firstly, restricted by the hard material of shell panels, the body shapes that the robot can imitate are limited, especially for the waist part. Secondly, signal transmission of the control system has a little delay. Thirdly, due to the narrow internal installation space, there is a certain restriction in the mechanism, which makes that the size range of the robot be smaller than the expected value.

\section{Conclusion}

In this paper, we present a design of a form-changing female fitting robot. The design of the mechanical structure and control system is introduced. Based on the design, a prototype is produced, and experiments show that it can imitate a range of female body types, which provides a promising solution for garment e-commerce and made-to-measure.

In the future, we will improve the form-changing female fitting robot from the following aspects:

(1) The scheme of model panels will be further optimized to make the robot have a more natural deformation. The design of model panels is the basis of mechanical structure and control system design. It determines the robot's simulation of the human body.

(2) An appropriate shell material should be chosen, so that the shell panels can bend and carry out the flexible change to ensure the fitting robot can simulate more types of human bodies.

(3) The mechanical structures need further refinement to improve its accuracy and flexibility and expand the deformation range.

(4) 3D scanning data of a real human will be used to directly drive the deformation of fitting robot, so that the fitting robot has the same shape as the real person.

\section{Acknowledgement}

We would like to thank Aobo Zhou, Lei Shi and Ke Ma for designing and producing the prototype of first generation of a male fitting robot, which paves a way for the work in the paper. This work was supported in part by National Science Foundation of China 61472355 and 51575481, Fundamental Research Funds for the Central Universities of China ZJUR041403, and Zhejiang Provincial Natural Science Foundation of China LY13F020003.

\section{References}

Abels A. and Kruusmaa M., Construction of a Female Shape-Changing Robotic Mannequin, Journal of Automation \& Control Engineering Vol.1, No.2(2013a), pp.132-134.

Abels A. and Kruusmaa M., Design of a shape-changing anthropomorphic mannequin for tailoring applications, Advanced Robotics (2009). ICAR 2009. International Conference on IEEE (2009), pp.1-6.

Abels A. and Kruusmaa M., Shape control of an anthropomotric tailoring robot mannequin, International Journal of Humanoid Robotics, Vol.10, No.2 (2013b), DOI: 10.1142/S0219843613500023.

AR Door Company's Homepage. (Online). Available: http://www.ar-door.com, (accessed on 19 May, 2015).

Baraff D. and Witkin A., Large steps in cloth simulation, Computer Graphics (SIGGRAPH 98 Proceedings) (2001), pp.43-54.

Baek S.Y. and Lee K., Parametric human body shape modeling framework for human-centered product design, Computer-Aided Design, Vol.44, No.1 (2012), pp.56-67.

Choi K.J. and Ko H.S., Stable but Responsive Cloth, ACM Trans. Graph. Vol.21, No.3 (2002), pp.604-611.

Cheverud J., Gordon C., et al, Anthropometric Survey of US Army Personnel: Correlation Coefficients and Regression Equations, Part 3 Simple and Partial Correlation Tables--Female, Natick, MA: US Army Natick Research, Development, and Engineering Center. AD (1988).

Fits.me Company's Homepage. (Online). Available: http://www.fits.me/, (accessed on 11 May, 2015).

Goldenthal R., Harmon D., Fattal R., Bcrcovier M. and Grinspun T., Efficient Simulation of Inextensible Cloth, ACM Transactions on Graphics, Vol.26, No.3 (2007):49.

Kim S. and Park C.K., Parametric body model generation for garment drape simulation, Fibers and Polymers, Vol.5, No.1(2004), pp.12-18. 
Lee Y.J., Yoon S.E., Oh S., Kim D. and Choi S., Multi-Resolution Cloth Simulation, Computer Graphics Forum, Vol.29, No.7 (2010), pp.2225-22232.

Metail Company's Homepage. (Online). Available: http://www.metail.com, (accessed on 13 May, 2015).

Peng S.X., Development of an interactive robotic mannequin for fashion industry, PHD Thesis, The Hong Kong Polytechnic University (2013).

Terzopoulost D., Platt J., Barr A. and Fleischert K., Elastically Deformable Models, Acm Siggraph Computer Graphics, Vol.21, No.4 (1987), pp.205-214.

Taylor P.J. and Shoben M.M., Grading for the fashion industry: The theory and practice, Stanley Thornes (1993).

Weil J., The Synthesis of Cloth Objects, ACM SIGGRAPH Computer Graphics, Vol.20, No.4 (1986), pp.49-54.

Wang C.C.L., Parameterization and parametric design of mannequins, Computer-Aided Design (2005).

Zhou C., Jin X.G. and Wang C.L., Shear Buckling and Dynamic Bending in Cloth Simulation, Computer Animation and Virtual Worlds, Vol.19, No.3 (2008), pp.493-503. 